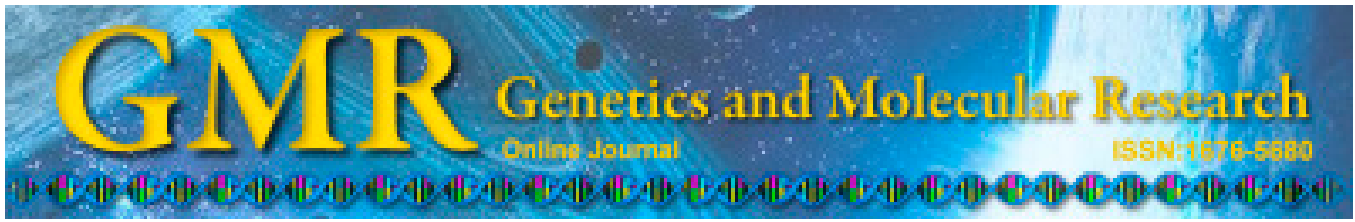

\title{
Fatty acid composition evaluation of edible parts of Eriocheir sinensis in intensive ponds of Gucheng waters
}

\author{
J.Y. Yu and P.S. Yan \\ College of Animal Science and Technology, Nanjing Agricultural University, \\ China \\ Corresponding author: P.S. Yan \\ E-mail: yanps@hotmail.com
}

Genet. Mol. Res. 14 (2): 5334-5345 (2015)

Received April 9, 2014

Accepted August 15, 2014

Published May 22, 2015

DOI http://dx.doi.org/10.4238/2015.May.22.4

\begin{abstract}
In Gucheng Lake, Jiangsu Province, China, randomly selected crabs were fed 2 diet types. Crab crude oil methyl ester analysis was performed using gas chromatography-mass spectrometry. Fatty acid compositions in the 3 edible parts of a crab - the hepatopancreas, gonad, and muscle - were analyzed. C16:1 and C18:2 were significantly higher in most commercial pellet feed-feeding crabs than in small trash fish-feeding crabs, while the opposite was observed for eicosapentaenoic and docosahexaenoic acids. Phytanic acid reached $1.93 \%$ in the hepatopancreas of small trash fish-feeding crabs. Furan fatty-acid-DiMe $(11,5)$ contents in the testes of small trash fish-feeding crabs was $1.49 \%$. These values were higher in male crabs than in female crabs. According to a standard ratio of 1:1:1 which meaning the saturated fathy acid (SFA), monounsaturated fatty acid (MUFA), and polyunsaturated fatty acid (PUFA) were 33.33 each, fatty acid structure analysis of crab edible parts showed that SFA:MUFA:PUFA of crab edible parts was 2.3-4.1:2.9-5.0:1.3-4.8. The highest muscle score was 29.53 in male trash fish-feeding crabs, and the lowest hepatopancreas score was -40.81 in female commercial pellet feed-feeding crabs. The
\end{abstract}


$\mathrm{n}-6 / \mathrm{n}-3$ ratio was $0.36-2.48$. Muscle ratio was the lowest in female commercial pellet feed-feeding crabs. Thus, small trash fish-feeding and commercial pellet feed-feeding crabs are healthy foods. Overall, for consumption, the males of small trash fish-feeding crabs were better than the females, the muscle was better than the gonads and hepatopancreas, and the testis was better than the ovary.

Key words: F-acid-DiMe $(11,5)$; Saturated fatty acid; Phytanic acid; Crab; Monounsaturated fatty acid; Polyunsaturated fatty acid

\section{INTRODUCTION}

The Chinese mitten crab (Eriocheir sinensis) is a traditional Chinese freshwater aquaculture product; it has good flavor as well as food value and is highly priced. Yangcheng Lake, Taihu Lake, and Gucheng Lake along the Yangtze River are the main Chinese crab-raising areas; in the consumer market, the Gucheng Lake crab is comparable to the Yangcheng Lake crab in the Jiangsu Province (Figure 1).

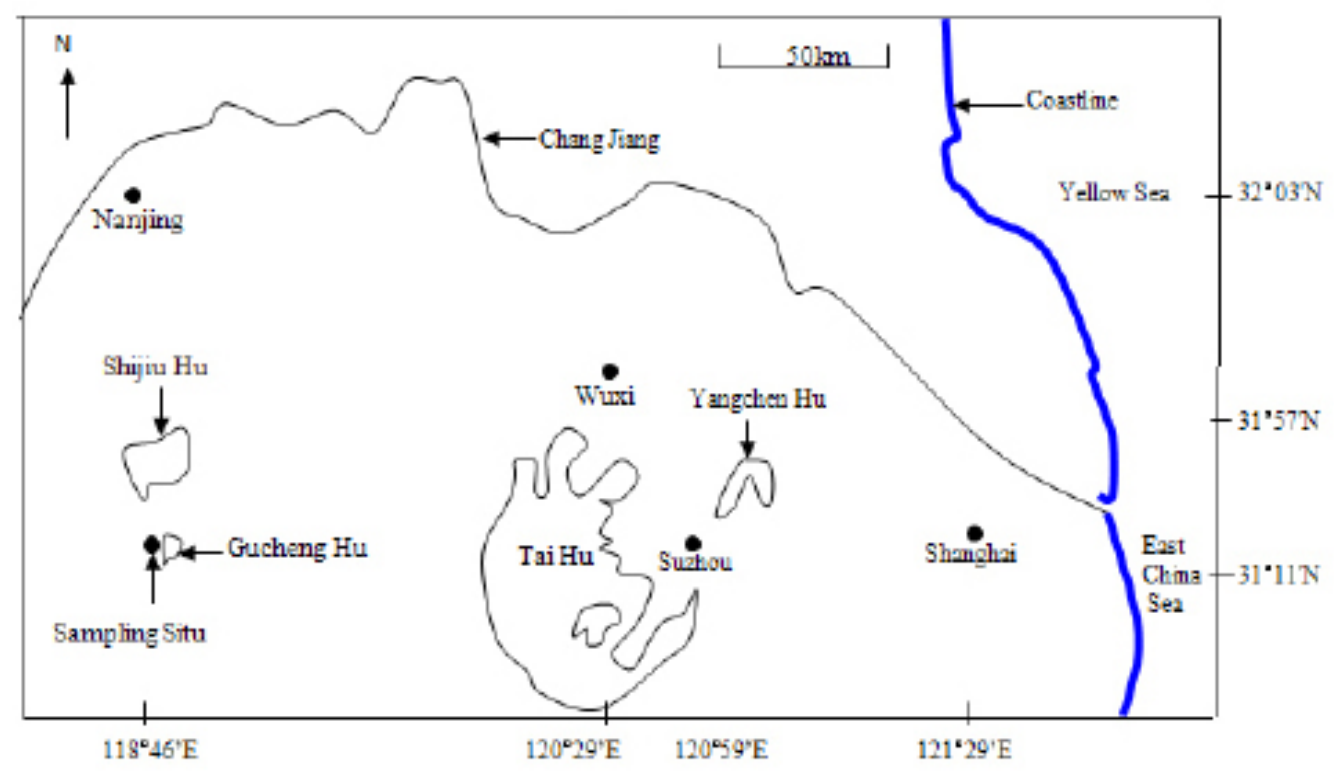

Figure 1. Primary crab waters along the Yangtze River and sampling locations of the Gucheng crab.

Feeding of trash fish is the common crab-farming method in the Jiangsu Province (Cai et al., 2012). With scarce resources of small trash fish and environmental pollution caused by farming, the trend of commercial formulated pellet feed in aquaculture feeding increases yearly. Therefore, assessing different feeding methods on crab quality is of certain economic value and social significance for maintaining the unique flavor and local crab- 
farming efficiency. Fat is the most important type of meat nutrient; the composition directly reflects the fatty acid composition of the food chain and affects the quality and flavor of food. Therefore, we fed crabs different diets to evaluate fatty acid composition in the edible parts of the crab.

\section{MATERIAL AND METHODS}

\section{Reagents}

Petroleum ether, hexane, potassium hydroxide, boron trifluoride methanol, dried over anhydrous sodium sulfate, sodium chloride, and 37 types of fatty acid methyl ester mixture standard products $(10 \mathrm{mg} / \mathrm{mL}, 1 \mathrm{~mL}$; Supelco, Bellefonte, PA, USA) were used in this study.

\section{Experimental instrument}

A Soxhlet extractor, rotary evaporator, accelerated solvent extractor, and 1200-L gas chromatography-mass spectrometry (GC-MS) devices were obtained from Varian Medical Systems (Palo Alto, CA, USA).

\section{Crab sampling}

From Feburary to December of 2009, trial crabs were reared in 6 samilar crabintensive ponds beside Gaochun county containing water originating from Gucheng Lake water in Jiangsu Province (Figure 1). The conditions and environment of the ponds were samilar, such as pond age, size and depth of the pool, larvae, feeding, grass, fertilizer and other farming model basically the same. Three ponds were fed with small trash fish, while the remaining 3 were fed with commercial crab pellet feed. In each pond, 2 male and 2 female crabs of the same size were randomly selected; a total of 24 crabs were transported to the lab and weighed. The hepatopancrease, gonads, and muscle were manually dissected (foot meat and brisket meat were mixed). Samples were stored at $-20^{\circ} \mathrm{C}$ until fatty acid composition analysis.

\section{Sample preparation analysis}

Based on the method of Chen and Zhang (2007), samples of approximately $0.5 \mathrm{~g}$ were weighed. Soxhlet methods were used to extract the fat, and vacuum rotary evaporated methods were used to obtain oil. Hexane was used to dissolve the crab oil; a KOH-methanol bath was used to complete saponification. A boron trifluoride diethyl ether-methanol bath was performed to complete methyl ester preparation. Hexane was used for fatty acid methyl ester extraction, and saturated saline mater was added to promote stratification. Hexane was used for dilution and was analyzed by GC-MS analysis.

\section{Experimental methods}

The PEG-20N polar quartz capillary column $(30 \mathrm{~m} \times 0.25 \mathrm{~mm} \times 0.25 \mu \mathrm{m})$ and 
GC-MS temperament were used to detect the relative quality of fatty acid content in crab tissue samples. GC-MS conditions were described previously by Chen and Zhang (2007). NIST 98 and Wiley 275 computers were used for the spectral library search. Peak identification of the fatty acids in the analyzed crab samples was carried out by the comparison with retention times and mass spectra of known standards. Compounds of MS spectra were compared with a standard spectrum spectral library to determine similarity and revert similarity; the results showed that more than 800 peak area similarity were included in the statistics.

\section{Data analysis}

SPSS 16.0 (SPSS, Inc., Chicago, IL, USA) was used to perform independent sample $t$-tests on the continuous data or 1-way analysis of variance. Data are reported as means \pm standard error. The significance of the average values for each group was compared. $\mathrm{P}<0.05$ was considered to be statistically significant, and $\mathrm{P}<0.01$ was considered to be extremely significant.

\section{RESULTS}

In this study, between small trash-feeding crab and commercial pellet feed-feeding crab, there was no significant difference in male and female crab body weight hepatopancreas weight and gonad weight but was significant in male and female crab hepatopancreas, gonad, muscle fatty acid composition (Table 1).

Table 1. Crab sampling specifications $(\mathrm{N}=6$, means $\pm \mathrm{SE})$.
\begin{tabular}{lrrrr}
\hline Items & \multicolumn{1}{l}{ Trash male } & Feed male & Trash female & Feed female \\
\hline Body weight (g/unit) & $191.51 \pm 6.24$ & $189.20 \pm 6.71$ & $141.36 \pm 4.63$ & $134.87 \pm 4.34$ \\
Hepatopancreas weight (g/unit) & $12.30 \pm 0.55$ & $13.42 \pm 0.59$ & $8.66 \pm 0.53$ & $8.05 \pm 0.41$ \\
Ovary weight (g/unit) & $6.27 \pm 0.33$ & $7.31 \pm 0.24$ & $17.48 \pm 0.90$ & $16.25 \pm 0.60$ \\
\hline
\end{tabular}

\section{Comparison of fatty acid composition of hepatopancreas}

Twenty-seven fatty acids were detected in the hepatopancreas. Compared with small trash-feeding crab, male hepatopancreas $\mathrm{C} 14: 0$ of commercial pellet feed-feeding crabs was significantly higher $(\mathrm{P}=0.025)$; phytanic acid decreased significantly $(\mathrm{P}<0.000)$. cyclopropane fatty acid (CPA) 2-hexyl $(\mathrm{P}=0.075), \mathrm{C} 17: 0(\mathrm{P}=0.082), \mathrm{C} 18: 2(\mathrm{P}=0.073)$, and $\mathrm{C} 20: 2$ $(\mathrm{P}=0.092)$ showed an increasing trend, while F-acid-DiMe (11,5) $(\mathrm{P}=0.067), \mathrm{C} 20: 1(\mathrm{P}$ $=0.089)$, and $\mathrm{C} 22: 0(\mathrm{P}=0.102)$ showed a decreasing trend. The hepatopancreas $\mathrm{C} 16: 0$ of female commercial pellet feed-feeding crabs significantly increased $(\mathrm{P}=0.049) ; \mathrm{C} 19: 0(\mathrm{P}=$ $0.042)$, and $\mathrm{C} 22: 1(\mathrm{P}=0.052)$ significantly decreased; $\mathrm{C} 20: 3(\mathrm{P}=0.003)$, Arachidonic $(\mathrm{P}=$ $0.001)$, eicosapentaenoic acid (EPA) $(\mathrm{P}<0.000), \mathrm{F}$-acid-DiMe $(11,5)(\mathrm{P}=0.005)$, and docosahexaenoic acid (DHA) $(\mathrm{P}<0.000)$ decreased significantly; $\mathrm{C} 14: 1(\mathrm{P}=0.086)$ and $\mathrm{C} 16: 1$ $(\mathrm{P}=0.08)$ showed an increasing trend, while $\mathrm{C} 20: 1(\mathrm{P}=0.072)$ showed a decreasing trend. Other fatty acids between male crabs and female crabs showed no differences (Figure 2). 

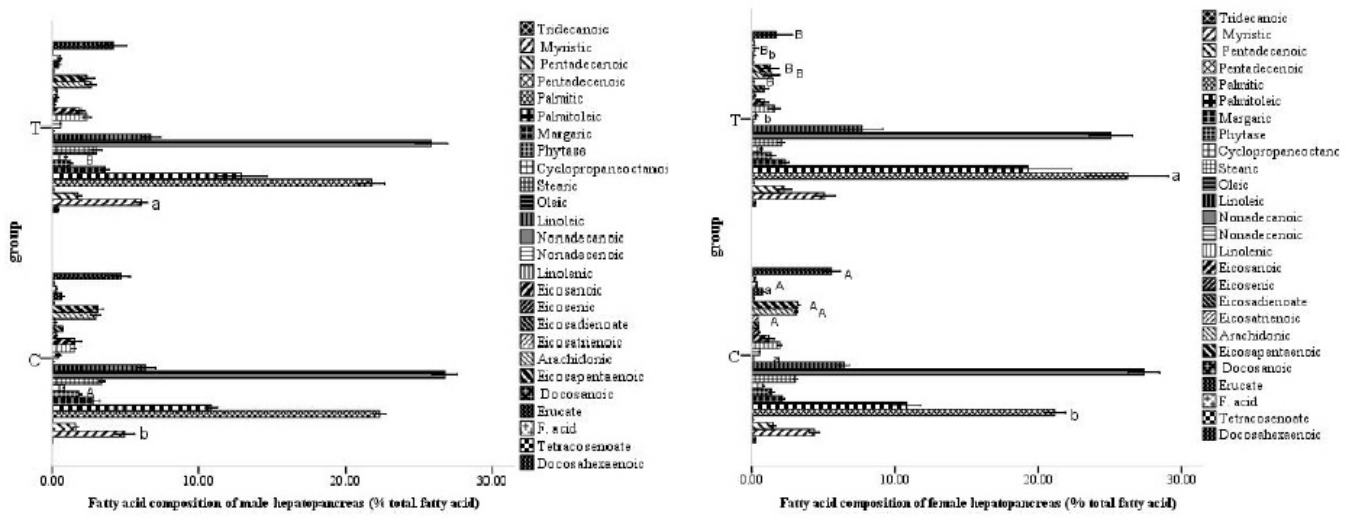

Figure 2. Fatty acid composition of crab hepatopancreas. The male and female crabs were compared between groups and analyzed using the independent samples test. Different lowercase letters at the top of the same column chart indicate significant differences $(\mathrm{P}<0.05)$. Different uppercase letters indicated a significant difference $(\mathrm{P}<$ $0.01)(\mathrm{N}=6$, means $\pm \mathrm{SE})$. Subsequent diagrams follow these guidelines as well.

\section{Comparison of fatty acid composition in gonad}

Twenty-one different fatty acids were detected in testis of the male crab; 22 different fatty acids were detected in the ovary of the female crab. C22:0, C22:1, CPA 2-octyl, and C24:1 contained in testis were not detected in ovaries; $\mathrm{C} 19: 1$ contained in the ovary was not detected in the testis. Compared with the small trash fish-feeding crab, F-acid-DiMe $(11,5)$ in the testis of the commercial pellet feed-feeding crab was significantly lower $(\mathrm{P}=0.035) ; \mathrm{C} 17: 0(\mathrm{P}=0.015)$ and $\mathrm{C} 18$ : $2(\mathrm{P}=0.015)$ was significantly higher; $\mathrm{C} 20: 2$ significantly increased $(\mathrm{P}=0.003)$, and $\mathrm{C} 22: 1(\mathrm{P}=$ $0.065)$ showed a decreasing trend. Phytanic acid $(\mathrm{P}=0.018), \mathrm{CPA}(\mathrm{P}=0.017), \mathrm{C} 18.2(\mathrm{P}=0.015)$, $\mathrm{C} 19.1(\mathrm{P}=0.034), \mathrm{C} 20.1(\mathrm{P}=0.011)$, and F-acid-DiMe $(11,5)(\mathrm{P}=0.035)$ in ovary of commercial pellet feed-feeding female crab was significantly decreased; $\mathrm{C} 14(\mathrm{P}=0.002), \mathrm{C} 20.5(\mathrm{P}=0.000)$, and $\mathrm{C} 22.6(\mathrm{P}=0.001)$ decreased very significantly; $\mathrm{C} 17(\mathrm{P}=0.023)$ significantly increased; $\mathrm{C} 16.1$ $(\mathrm{P}=0.007), \mathrm{C} 18.2(\mathrm{P}=0.005)$, and $\mathrm{C} 20.2(\mathrm{P}=0.003)$ very significantly increased; $\mathrm{C} 20.2(\mathrm{P}=0.07)$
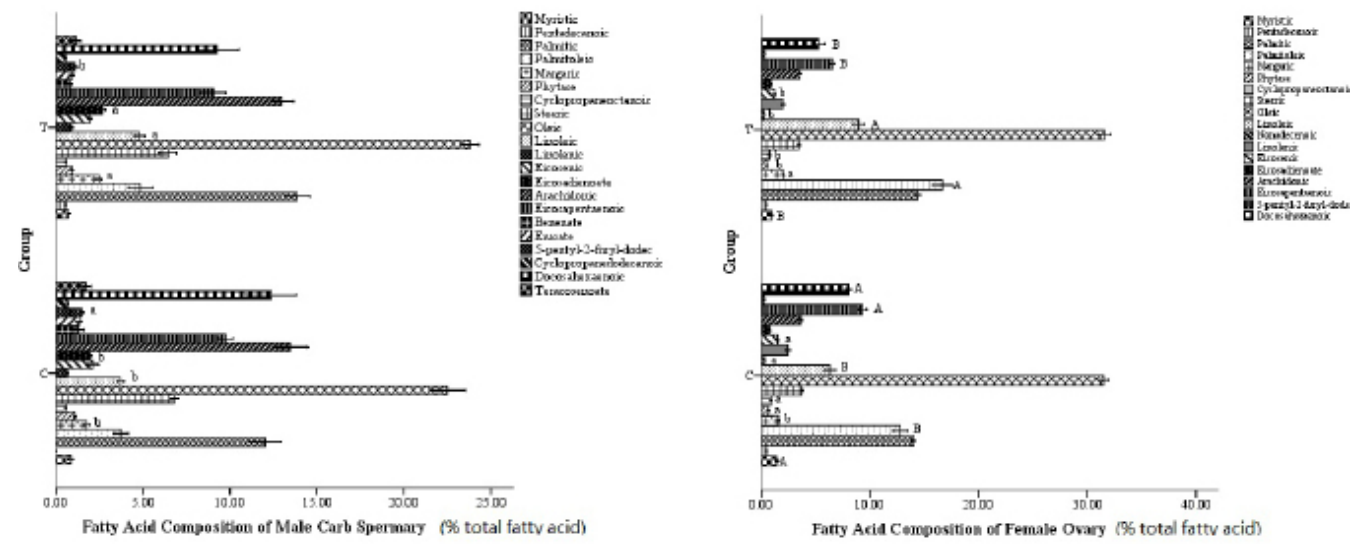

Figure 3. Fatty acid composition in a crab gonad. 
showed an increasing trend; $\mathrm{C} 18: 0(\mathrm{P}=0.084)$ and $\mathrm{C} 22.1(\mathrm{P}=0.065)$ showed a decreasing trend. Other fatty acids between male and female crabs showed no differences (Figure 3 ).

\section{Comparison of fatty acid composition in muscle}

Nineteen different fatty acids were detected in muscle samples. Compared with the small fish-feeding crab, the male commercial pellet feed-feeding crab contained significantly less DHA $(\mathrm{P}=0.032) ; \mathrm{C} 17: 0(\mathrm{P}=0.044)$ significantly increased, $\mathrm{C} 18: 2(\mathrm{P}=0.009)$ extremely significant increased, $\mathrm{C} 14: 0(\mathrm{P}=0.059)$ and EPA $(\mathrm{P}=0.074)$ showed a decreased trend. Muscle DHA of the female crab decreased significantly $(\mathrm{P}=0.006), \mathrm{C} 18: 1(\mathrm{P}=0.014)$ and $\mathrm{C} 18: 2(\mathrm{P}=0.007)$ very significantly increased, CPA $(\mathrm{P}=0.095)$ and F-acid-DiMe $(11,5)(\mathrm{P}=0.102)$ showed decreased trend. Other fatty acids between male and female crabs showed no differences (Figure 4).
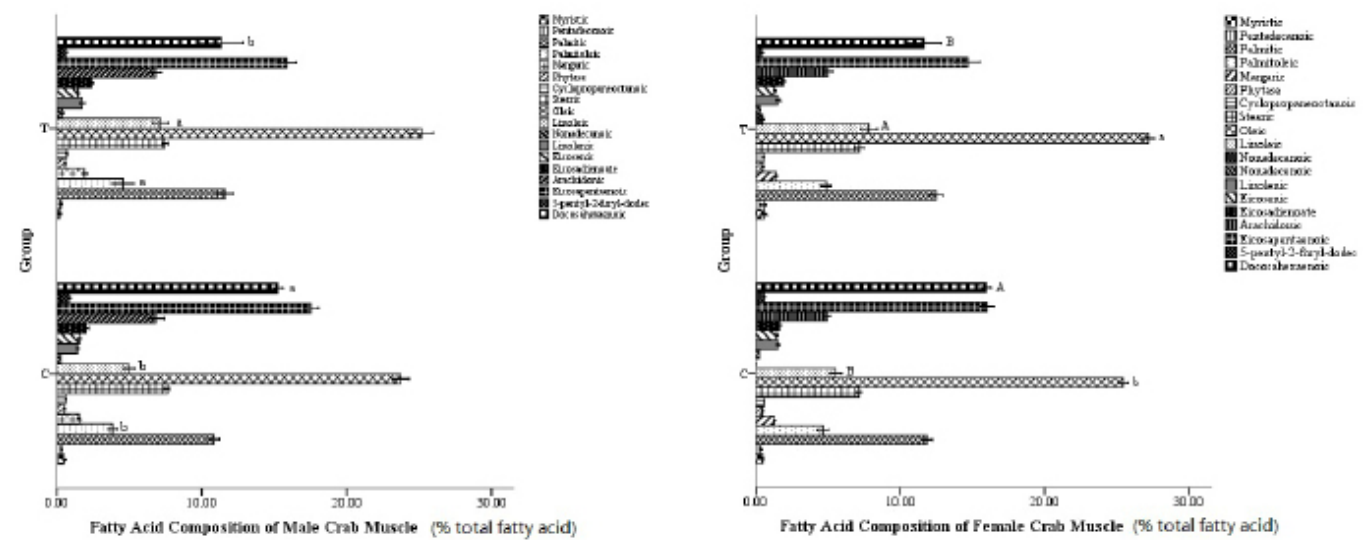

Figure 4. Fatty acid composition in a crab muscle.

\section{DISCUSSION}

To assess the quality of crab fatty acids, we analyzed saturated fatty acid (SFA): monounsaturated fatty acid (MUFA): polyunsaturated fatty acid (PUFA), n-6/n-3 and EPA, DHA, phytanic acid, and F-acid composition.

\section{Structural analysis of fatty acid composition of crab edible parts}

\section{SFA:MUFA:PUFA comparison}

The Food and Agriculture Organization of the United Nations and World Health Organization (2008) recommend that the SFA, MUFA, PUFA ratio should be 1:1:1 in the diet. In this study, SPA:MUFA:PUFA in the edible portion of the crab were 2.3-4.1:2.9-5.0:1.3-4.8. SFA was the highest in the hepatopancreas, MUFA was the highest in male commercial pellet feed-feeding crab, and PUFA was the highest in muscle. In the hepatopancreas, PUFA in the female commercial pellet feed-feeding crab was significantly lower than that in male and female small trash fish-feeding crabs and male commercial pellet feed-feeding crabs $(\mathrm{P}<0.01)$; 
other differences were not significant.

In the gonads, overall SFA and PUFA content in male and female small trash fishfeeding crabs was significantly higher than that in male and female commercial pellet feedfeeding crabs $(\mathrm{P}<0.01)$; MUFA in male and female small trash fish-feeding crabs was significantly lower than that in commercial pellet feed-feeding crabs $(\mathrm{P}<0.01)$. In the muscle, overall differences in SFA, MUFA, and PUFA between groups were not significant (Table 2).

Table 2. Structural analysis of fatty acid composition of crab edible parts $(\mathrm{N}=6$, means $\pm \mathrm{SE})$.

\begin{tabular}{|c|c|c|c|c|c|c|c|c|}
\hline \multirow[t]{2}{*}{ Items } & \multicolumn{2}{|c|}{ Male crab composition* } & \multicolumn{2}{|c|}{ Female crab composition* } & \multicolumn{2}{|c|}{ Male crab score } & \multicolumn{2}{|c|}{ Female crab score } \\
\hline & Trash & Feed & Trash & Feed & Trash & Feed & Trash & Feed \\
\hline \multicolumn{9}{|l|}{ Hepato } \\
\hline SFA & $38.70 \pm 0.40$ & $37.01 \pm 1.45$ & $35.36 \pm 1.00$ & $41.27 \pm 2.34$ & -5.37 & -3.68 & -2.03 & -7.94 \\
\hline MUFA & $40.50 \pm 0.82^{\mathrm{b}}$ & $42.25 \pm 1.76$ & $43.34 \pm 0.82$ & $45.80 \pm 1.52^{\mathrm{a}}$ & -7.17 & -8.92 & -10.01 & -12.47 \\
\hline PUFA & $20.81 \pm 0.67^{\mathrm{A}}$ & $20.73 \pm 1.89^{\mathrm{A}}$ & $21.29 \pm 0.96^{\mathrm{A}}$ & $12.93 \pm 2.14^{\mathrm{B}}$ & -12.52 & -12.6 & -12.04 & -20.4 \\
\hline Total & & & & & -25.06 & -25.2 & -24.08 & -40.81 \\
\hline \multicolumn{9}{|l|}{ Gonad } \\
\hline SFA & $26.58 \pm 1.30^{\mathrm{a}}$ & $27.66 \pm 0.72^{\mathrm{A}}$ & $23.23 \pm 0.32^{\mathrm{bB}}$ & $23.11 \pm 0.29^{\mathrm{bB}}$ & 6.75 & 5.67 & 10.1 & 10.22 \\
\hline MUFA & $31.48 \pm 1.21^{\mathrm{B}}$ & $32.68 \pm 0.89^{\mathrm{B}}$ & $46.22 \pm 0.55^{\mathrm{A}}$ & $49.72 \pm 0.84^{\mathrm{A}}$ & 1.85 & 0.65 & -12.89 & -16.39 \\
\hline PUFA & $41.94 \pm 2.43^{\mathrm{A}}$ & $39.66 \pm 1.19^{\mathrm{A}}$ & $30.55 \pm 0.48^{\mathrm{B}}$ & $27.17 \pm 0.76^{\mathrm{B}}$ & 8.61 & 6.33 & -2.78 & -6.16 \\
\hline Total & & & & 17.21 & 12.65 & -5.57 & -12.33 & \\
\hline \multicolumn{9}{|l|}{ Muscle } \\
\hline SFA & $22.77 \pm 0.42$ & $23.43 \pm 0.61$ & $22.71 \pm 0.23$ & $23.66 \pm 0.51$ & 10.56 & 9.9 & 10.62 & 9.67 \\
\hline MUFA & $29.13 \pm 0.82^{\mathrm{B}}$ & $31.24 \pm 1.48$ & $31.60 \pm 0.39$ & $33.66 \pm 0.45^{\mathrm{A}}$ & 4.2 & 2.09 & 1.73 & -0.33 \\
\hline PUFA & $48.10 \pm 1.13^{\mathrm{a}}$ & $45.33 \pm 1.88$ & $45.69 \pm 0.47$ & $42.68 \pm 0.85^{\mathrm{b}}$ & 14.77 & 12 & 12.36 & 9.35 \\
\hline Total & & & & & 29.53 & 23.99 & 24.71 & 18.69 \\
\hline
\end{tabular}

*One-way analysis of variance.

Excessive SFA and high MUFA can cause an energy imbalance and weight gain within the body, inducing insulin resistance and other nutritional diseases. PUFA, particularly n-3 PUFA, can eliminate this resistance (Lovejoy, 2012). Therefore, we set SFA + MUFA + PUFA $=100$ scores, and the SFA, MUFA, and PUFA were averaged of 33.33 each for a standard ratio of 1:1:1.

More than 1 score PUFA was considered to be positive, while more than 1 score SFA and MUFA were considered to be negative. According to Table 2, the SFA:MUFA:PUFA values were used to assess fatty acid structure scores of crab edible parts. The results showed that the muscle of the male small trash fish-feeding crab was as high as 29.53; the hepatopancreas of female commercial pellet feed-feeding crab was as low as -40.81 (Table 2). This suggested that after SFA:MUFA:PUFA comparison, the small trash fish feeding was nutritionally superior to commercial pellet feed feeding crabs, and the male crabs were better than female crabs, muscle was better than the gonads, and gonads were better than the hepatopancreas.

\section{n-6/n-3 ratio comparison}

The biological activity and physiological functions of PUFA are affected by the n-6/n-3 ratio. The Food and Agriculture Organization of the United Nations and World Health Organization recommended that the dietary $n-6 / n-3$ should be $5: 1$ to $10: 1$. In this study, the $n-6 / n-3$ ratio of crab edible parts was $0.36-2.48$, which was better than the ratio of $5: 1$. We set that the $n-6 / n-3$ of 0:1 was used as a score of 200, 5:1 as 100 , and 10:1 as 0 , and the function score equation of $n$ 6/n-3 ratio was as follows: $n-6 / n-3$ score $=-20 \times(n-6 / n-3)+200$. The results showed that $n-6 / n-3$ scores were 150.43-192.73; these values were better than the 5:1 ratio of 100 points. The muscle of female small trash fish-feeding crabs was the highest; the hepatopancreas of the female com- 
mercial pellet feed-feeding crab was of lowest quality (Table 3 ). This suggests that, based on the ratio of $n-6 / n-3$, the crab edible parts contain a high-quality fatty acid structure, the muscle was better than the gonads, and the gonads were better than the hepatopancreas.

Table 3. Score evaluation of fatty acids with $n-6 / n-3$ ratio in crab edible parts (score $=-20 \times$ ratio +200$)$.

\begin{tabular}{|c|c|c|c|c|c|c|c|c|}
\hline \multirow[t]{2}{*}{ Items } & \multicolumn{4}{|c|}{ n- $6 / n-3$ ratio } & \multicolumn{4}{|c|}{$n-6 / n-3$ score } \\
\hline & Trash male & Feed male & Trash female & Feed female & Trash male & Feed male & Trash female & Feed female \\
\hline Hepatopancreas & 1.19 & 1.41 & 0.97 & 2.48 & 176.3 & 171.83 & 180.67 & 150.43 \\
\hline Gonad & 0.85 & 1.08 & 0.55 & 1 & 183 & 178.47 & 189.03 & 180.03 \\
\hline Muscle & 0.41 & 0.58 & 0.36 & 0.54 & 191.9 & 188.33 & 192.73 & 189.23 \\
\hline
\end{tabular}

Major fatty acids and special fatty acid composition analysis of crab edible parts

\section{C16:1, C18:2, EPA, and DHA comparison}

In this study, C16:1 and C18:2 content in commercial pellet feed-feeding crabs were significantly higher than in small trash fish-feeding crabs. EPA and DHA in female commercial pellet feed-feeding crabs were mainly significantly lower than in small trash fish-feeding crabs (Table 4). By analyzing the hepatopancreas, gonad, and muscle fatty acid composition of Yangcheng Lake river Eriocheir sinensis (Chen and Zhang, 2007), female Yangcheng lake and pool Eriocheir sinensis (Kong et al., 2012), Portunus pelagicus (Wu et al., 2010), Carcinus mediterraneus (Cherif et al., 2008), Callinectes sapidus (Çelik et al., 2004), and Paralithodes and Chionoecetes (Latyshev et al., 2009), the results show that the relative mass of fat differed in different species, different origin, and different breeding patterns (Table 4). High MUFA caused an energy imbalance of the inner environment (Lovejoy, 2012); a high intake of C18:2 and low intake of MUFA were associated with the incidence of breast cancer (Goodstine, 2003). DHA and EPA can lower blood pressure and have antithrombotic and antitumor effects, as well as prevent heart disease and improve physiological functions. Increased DHA intake can significantly reduce breast cancer risk in women (Maillard et al., 2002). In the 5 types of crabs, the relative masses of C16:1 and C18:2 in the crab hepatopancreas were high, the overall EPA was low, and the overall DHA was in between these values. This suggests that in these crab species, lower C16:1 and C18:2 and higher EPA and DHA are better for human health. The quality was below that of Paralithodes and Chionoecetes and greater than that of Callinectes sapidus.

\section{Phytanic acid and furan fatty acid comparison}

We detected the phytanic acid, CPA, F-acid, and other fatty acids. These fatty acids showed some differences between groups and organizations (Table 5).

\section{Phytanic acid}

In this study, phytanic acid (hexadecanoic acid 3,7,11,15-tetramethyl) was detected in the samples of the edible parts of crab (Figure 5). Their relative qualities were $0.46-1.93 \%$ (Table 5). The quality of commercial pellet feed-feeding crabs was lower than that of small trash fish-feeding crabs. Chen and Zhang (2007) reported that $1.31 \%$ phytanic acid was detected in the hepatopancreas of Yangcheng Lake crabs. 


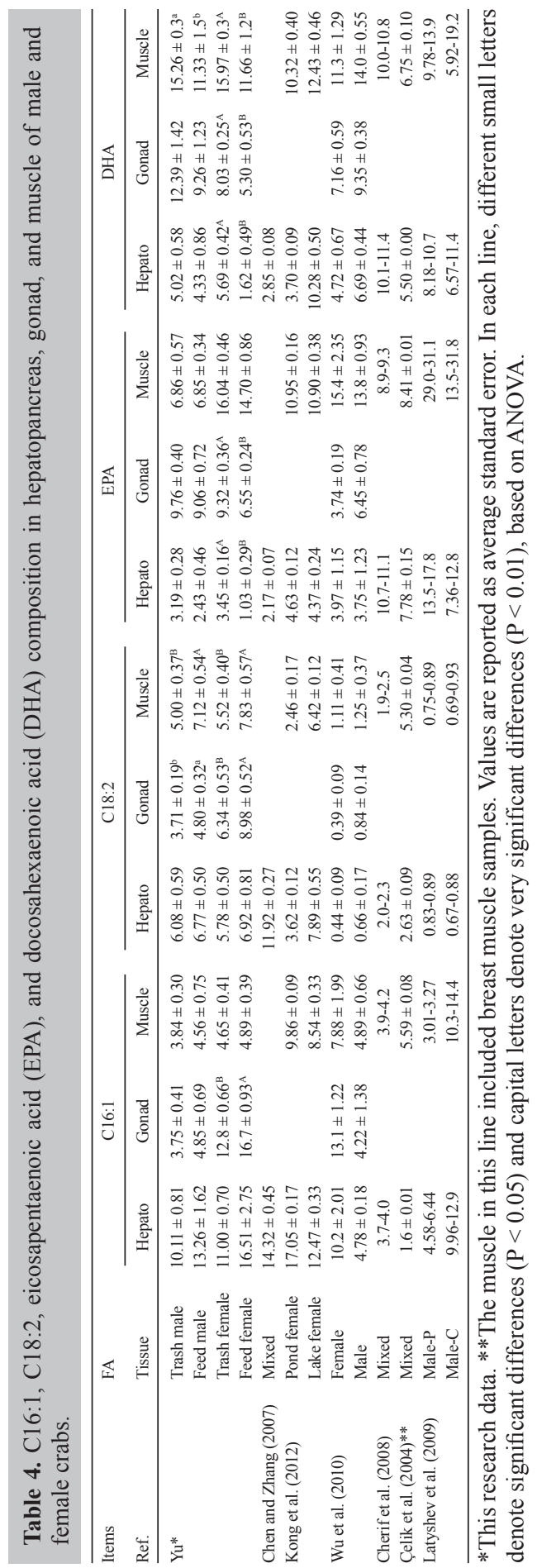


Table 5. Phytanic acid and furan fatty-acid compositions in the crab edible parts.

\begin{tabular}{|c|c|c|c|c|}
\hline Items & Trash male crab & Feed male crab & Trash female crab & Feed female crab \\
\hline \multicolumn{5}{|l|}{ Phytanic acid } \\
\hline Hepatopancreas & $1.93 \pm 0.08^{\mathrm{A}}$ & $1.21 \pm 0.11^{\mathrm{B}}$ & $1.38 \pm 0.13$ & $1.65 \pm 0.44$ \\
\hline Gonad & $1.09 \pm 0.07$ & $0.93 \pm 0.07$ & $0.70 \pm 0.04^{\mathrm{a}}$ & $0.57 \pm 0.03^{\mathrm{b}}$ \\
\hline Muscle & $0.53 \pm 0.43$ & $0.57 \pm 0.08$ & $0.46 \pm 0.02$ & $0.48 \pm 0.05$ \\
\hline \multicolumn{5}{|c|}{ fatty-acid-DiMe $(11,5)$} \\
\hline Hepatopancreas & $0.92 \pm 0.16$ & $0.55 \pm 0.09$ & $0.66 \pm 0.07^{\mathrm{A}}$ & $0.14 \pm 0.14^{\mathrm{B}}$ \\
\hline Gonad & $1.49 \pm 0.12^{\mathrm{a}}$ & $1.10 \pm 0.11^{\mathrm{b}}$ & $0.30 \pm 0.06$ & $0.32 \pm 0.01$ \\
\hline Muscle & $0.85 \pm 0.06$ & $0.63 \pm 0.12$ & $0.57 \pm 0.06$ & $0.41 \pm 0.07$ \\
\hline
\end{tabular}

Values are reported as average standard error. In each line, different small letters denote significant differences $(\mathrm{P}<$ $0.05)$ and capital letters denote very significant differences $(\mathrm{P}<0.01)$, based on ANOVA.

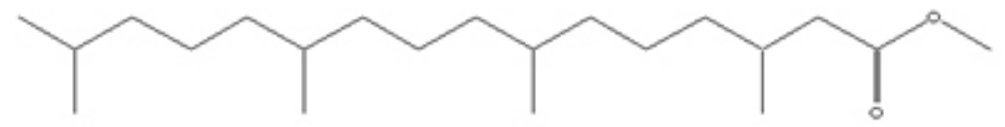

Figure 5. Phytanic acid structure.

Phytanic acid is widely present in many foods in small amount; for example, the phytanic acid content in fish oil can reach $700 \mathrm{mg} / 100 \mathrm{~g}$ (Brown et al., 1993). Phytanic acid content is $296 \mathrm{mg} / 100 \mathrm{~g}$ in German Dennree whole organic milk and $176 \mathrm{mg} / 100 \mathrm{~g}$ in Alpine ordinary milk. It can reach $211 \mathrm{mg} / 100 \mathrm{~g}$ in organic raw milk butter and $141 \mathrm{mg} / 100 \mathrm{~g}$ in ordinary butter (Vetter and Schröder, 2010). After comparison, river crab contains a relatively high amount of phytanic acid. Phytanic acid is oxidized in the peroxisomes and mitochondria. After $\alpha$-oxidization, formic acid and pristine are formed; subsequently, pristane acid continues to go through oxidative metabolism via the $\beta$-oxidation pathway. In phytanic acid $\alpha$-oxidation disorders, large amounts of phytanic acid accumulate in the human body, causing Refsum's disease (also known as phytanic acid storage disease) (Wanders, 2003). A previous study showed that phytanic acid had cytotoxic effects on the mitochondria and stellate cells in the rat brain (Schönfeld et al., 2006). However, both phytanic and pristane acids play a regulatory role in adipocyte differentiation and polyester, glucose, and lipid metabolism (Heim, 2002); it can promote fatty acid $\beta$-oxidation and significantly lower liver triglyceride and plasma fatty acids (Gloerich et al., 2007). In this study, both commercial pellet feed-feeding and small trash fish-feeding crabs contained high levels of phytanic acid, suggesting that crab is similar to other high phytanic acid foods and may have potential health functions.

\section{F-acid}

In this study, furan fatty acids 11-(3,4-dimethy-5-pentyl-2-furyl)-dodecanoic acid, abbreviated as F-acid-DiMe $(11,5)$ or F-acid, was detected in the edible parts of crabs (Figure 6); they were higher in small trash fish-feeding crabs than in commercial pellet feedfeeding crabs, and higher in male crabs than in female crabs, which has not been reported previously. 


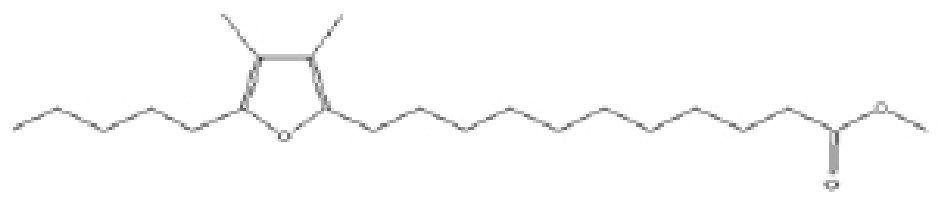

Figure 6. DiMe $(11,5)$ structure.

Multiple F-acids exist in nature; the crude oil of squid liver contains $0.52 \%$ of DiMe $(11,5)$ (Scrimageour, 1977). In the Turkmenistan region, Rhithropanopeus harrisii was found to contain 5 different F-acids, while DiMe $(11,5)$ accounted for $0.19 \%$ (Dembitsky and Rezanka, et al., 1996). In the spring and summer seasons, Merluccius merluccius contains up to $30 \mathrm{mg} / 100 \mathrm{~g}$ DiMe $(11,5)$, while Scomber scombrus contains $0.1 \mathrm{mg} / 100 \mathrm{~g}$ (Pacetti et al., 2010). F-acids have hydroxyl radical removal and antioxidant effects, can prevent oxidation of linoleic acid, and may have antitumor effects (Okada et al., 1996). Japanese diets are rich in fish oil or fish because of the presence of n-3 PUFA and F-acids (Spiteller, 2007). This suggests that F-acids are similar to EPA, DHA, and other fatty acids with functional effects. In this study, the testes of small trash fish-feeding crabs contained up to $1.49 \%$ fat content, while that of commercial pellet feed-feeding crabs was up to $1.10 \%$. This suggests that the testes fatty acids may have unique health functions.

\section{CONCLUSIONS}

In this study, lipid fatty SFA:MUFA:PUFA, and n-6/n-3 of edible parts showed differences between small trash fish-feeding crabs and commercial pellet feed-feeding crabs. C16:1, C18:2, EPA, DHA, phytanic acid, F-acid, and several other key fatty acids differed between groups. Small trash fish-feeding crabs and commercial pellet feed-feeding crabs are both healthy foods and recommended for consumption. However, small trash fish-feeding crabs were better than commercial pellet feed-feeding crabs. Overall, the male was better than the female, the muscle was better than the gonads, the gonads were better than the hepatopancreas, and the testes were better than the ovaries.

\section{REFERENCES}

Brown JP, Mei G, Gibberd FB, Burston D, et al. (1993). The determination of phytanic acid and phytol in certain foods and the application of this knowledge to the choice of suitable convenience foods for patients with Refsum's disease. J. Hum. Nutr. Diet. 61: 295-305.

Cai CF, Gu XH, Huang HZ, Dai XY, et al. (2012). Water quality, nutrient budget, and pollutant loads in Chinese mitten crab (Eriocheir sinensis) farms around East Taihu Lake. Chin. J. Oceanol. Limnol. 30: 29-36.

Çelik M, Türeli C, Çelik M, Yanai Y, et al. (2004). Fatty acid composition of the blue crab (Callinectes sapidus, Rathbun, 1896) in the north eastern Mediterranean. Food Chem. 88: 271-273.

Chen DW and Zhang M (2007). Non-volatile taste active compounds in the meat of Chinese mitten crab (Eriocheir sinensis). Food Chem. 104: 1200-1205.

Cherif S, Frikha F, Gargouri Y and Miled N (2008). Fatty acid composition of green crab (Carcinus mediterraneus) from the Tunisian Mediterranean coasts. Food Chem. 111: 930-933.

Dembitsky VM and Rezanka T (1996). Furan fatty acids of some brackish invertebrates from the Caspian Sea. Comp. Biochem. Physiol. 114: 317-320.

FAO/WHO (2008). Fats and fatty acids in human nutrition. Proceedings of the Joint FAO/WHO Expert Consultation, 77-82. 
Gloerich J, van den Brink DM, Ruiter JP, van Viles N, et al. (2007). Metabolism of phytol to phytanic acid in the mouse, and the role of PPAR $\alpha$ in its regulation. J. Lipid Res. 48: 77-85.

Goodstine SL, Zheng T, Holford TR, Ward BA, et al. (2003). Dietary (n-3)/(n-6) fatty acid ratio: Possible relationship to premenopausal but not postmenopausal breast cancer risk in US women. J. Nutr. 133: 1409-1414.

Heim M, Johnson J, Boess F, Bendik I, et al. (2002). Phytanic acid, a natural peroxisome proliferator-activated receptor (PPAR) agonist, regulates glucose metabolism in rat primary hepatocytes. FASEB J. 16: 718-720.

Kong L, Cai CF, Ye Y, Chen D, et al. (2012). Comparison of non-volatile compounds and sensory characteristics of Chinese mitten crabs (Eriocheir sinensis) reared in lakes and ponds: Potential environmental factors. Aquaculture 364: 96-102.

Latyshev NA, Kasyanov SP, Kharlamenko VI and Svetashev VI (2009). Lipids and of fatty acids of edible crabs of the north-western Pacific. Food Chem. 116: 657-661.

Lovejoy JC (2012). The influence of dietary fat on insulin resistance. Curr. Diab. Rep. 2: 435-440.

Maillard V, Bougnoux P, Ferrari P, Jourdan ML, et al. (2002). N-3 and N-6 fatty acids in breast adipose tissue and relative risk of breast cancer in a case-control study in Tours, France. Int. J. Cancer 98: 78-83.

Okada Y, Kanedo M and Okajima H (1996). Hydroxyl radical scavenging activity of naturally furan fatty acids. Biol. Pharm. Bull. 19: 1607-1610.

Pacetti D, Alberti F, Boselli E and Frega NG (2010). Characterisation of furan fatty acids in Adriatic fish. Food Chem. 122: 209-215.

Schönfeld P, Kahlert S and Reiser G (2006). A study of the cytotoxicity of branched-chain phytanic acid with mitochondria and rat brain astrocytes. Exp. Gerontol. 41: 688-696.

Scrimageour CM (1997). Quantitative analysis of furanoid fatty acids in crude and refined cod liver oil. J. Am. Oil Chem. Soc. 54: 210-211.

Spiteller G (2007). The important role of lipid peroxidation processes in aging and age dependent diseases. Mol. Biotechnol. 37: 5-12.

Vetter W and Schröder M (2010). Concentrations of phytanic acid and pristanic acid are higher in organic than in conventional dairy products from the German market. Food Chem. 119: 746-752.

Wanders RJ, Jansen GA and Lloyd MD (2003). Phytanic acid alpha-oxidation, new insights into an old problem: a review. Biochim. Biophys. Acta 1631: 119-135.

Wu XG, Zhou B, Cheng YX, Zeng CS, et al. (2010). Comparison of gender differences in biochemical composition and nutritional value of various edible parts of the blue swimmer crab. J. Food Comp. Anal. 23: 154-159. 